\title{
Nonlinear Noise of Low Transmission Penalty Dual-Stage Discrete Raman Amplifier
}

\author{
Lukasz Krzczanowicz, Md. Asif Iqbal, Ian Phillips, M. A. Z. Al-Khateeb, Mingming Tan, Paul Harper \\ and Wladek Forysiak
}

\begin{abstract}
We experimentally characterise the linear and nonlinear performance of a $>70 \mathrm{~nm}$, dual-stage, $19.5 \mathrm{~dB}$ average net gain discrete Raman amplifier using different nonlinear fibres in the second stage. We propose an architecture built with a combination of IDF and SMF, and compare its performance with amplifiers built with conventionally used nonlinear fibre types (IDF-IDF, IDF-DCF). The measured FWM product power shows the IDF-SMF architecture to generate less nonlinear interference when compared to other schemes. We test the amplifiers with $5 \times 120 \mathrm{~Gb} / \mathrm{s}$ DP-QPSK WDM signals in a recirculating loop at 10 recirculations of $93.4 \mathrm{~km}$ SMF fibre, where the power sweep shows up to $2 \mathrm{~dB}$ optimum launch power difference, with the maximum $Q^{2}$ factor varying by up to $1.6 \mathrm{~dB}$. Using the optimum transmission point we measure a $Q^{2}=8.8 \mathrm{~dB}$ at 35 recirculations of $93.4 \mathrm{~km}$ transmission $(3269 \mathrm{~km})$ with the proposed IDF-SMF scheme, which is $>460 \mathrm{~km}$ further than the other tested architectures. All characterised schemes performed similarly in the linear noise regime.
\end{abstract}

Index Terms-Discrete Raman Amplification, Broadband Transmission, Nonlinearity Characterisation.

\section{INTRODUCTION}

$\mathrm{R}$ AMAN amplification is a technique that can be used to increase fibre transmission capacity by enhancing the bandwidth of the transmitted signal which is currently limited by the commonly used erbium doped fibre amplifiers (EDFA). The bandwidth of those devices is limited to $\sim 40 \mathrm{~nm}$ with a possibility for doubling by combining EDFAs operating in different bands, but such an approach generates a "bandwidth gap" introducing excess losses due to band splitters. Raman amplification on the other hand provides seamless broad bandwidth gain [1] with fully configurable gain profile. By using multiple pump lasers it is possible to scale the bandwidth to over $100 \mathrm{~nm}$ [2], however this can be costly as achieving a flat gain spectrum requires pumping at many wavelengths [3]. The majority of deployed Raman amplifiers employ distributed amplification, which by using the transmission fibre as the gain medium itself improves the optical signal to noise ratio (OSNR) and thus directly increases the achievable transmission distance [1]. This technique however requires low loss between terminal equipment and transmission fibre, and high optical pump

Manuscript received xxxx, 2018; revised xxxx, 2018; accepted xxxx, 2018. Date of publication xxxx, 2018; date of current version xxxxx, 2018.

This work was funded by UK EPSRC project EP/M009092/1 (ToM3). We acknowledge the industrial support from II-VI. Corresponding author: Lukasz Krzczanowicz (1.krzczanowicz@aston.ac.uk). powers to be launched into the transmission fibre, which can be disadvantageous due to operational restrictions and laser safety.

Discrete Raman amplification (DRA), which by design uses a separate fibre as a gain medium overcomes these limitations, but at the expense of no improvement of the OSNR. Most reported discrete Raman amplifiers use dispersion compensating fibre (DCF) as the gain medium [4]. Other types of Raman gain fibre: highly nonlinear fibre (HNLF), dispersion shifted fibre (DSF) and inverse dispersion fibre (IDF) have also been investigated [5]. Choosing the right fibre type is an important factor because while a smaller core area results in higher gain, their high nonlinear index can result in Kerrinduced nonlinear penalties due to self-phase modulation (SPM) induced nonlinear phase shift (NPS), cross-phase modulation (XPM) and four-wave mixing (FWM) [5-7].

The dual-stage DRA approach is a concept that has been shown to increase the overall performance of a discrete amplifier [8]. Having two stages instead of one with an optical isolator in between them limits double Rayleigh scattering (DRS) induced multi-path interference (MPI) crosstalk, enabling an increase in single stage limited maximum gain [9].

In this paper, for the first time to our knowledge, we experimentally characterise the linear and nonlinear performance of $>70 \mathrm{~nm}, 19.5 \mathrm{~dB}$ gain dual-stage amplifier designs built with different fibre types (IDF, DCF, SMF) in the second stage and identify an IDF10km-SMF10km configuration as the one with lowest nonlinear transmission penalty. The first stage is built with $10 \mathrm{~km}$ of IDF, which was chosen because of its optimal trade-off between noise figure (NF), nonlinear impairments and gain efficiency compared with DCF and SMF [6]. We use $10 \mathrm{~km}$ of SMF, $5 \mathrm{~km}$ and $10 \mathrm{~km}$ of DCF and $7.5 \mathrm{~km}$ of IDF in the second stage, and compare the performance of all four architectures in terms of gain, noise figure and FWM product power, where we measure a $3.3 \mathrm{~dB}$ power difference in integrated FWM product between the best (IDF10km-SMF10km) and worst (IDF10km-DCF10km) performing setup. Finally, we characterise the performance of dual-stage DRA in a recirculating loop with the use of $5 \times 120 \mathrm{~Gb} / \mathrm{s}$ DP-QPSK signals at 10 spans of $93.4 \mathrm{~km}$ of SMF. We reach a distance of $3269 \mathrm{~km}\left(\mathrm{Q}^{2}=8.8 \mathrm{~dB}\right)$ with the use of our IDF-SMF amplifier which is twice as far as the worst

The authors are with Aston Institute of Photonics Technologies, Aston University, Birmingham, B4 7ET, UK.

Original data for this work is available through Aston Research Explorer (https://doi.org/10.17036/researchdata.aston.ac.uk.00000373). 
performing configuration, IDF-DCF10km. We ascribe this improvement to its reduced sensitivity to signal power and superior performance in the nonlinear regime.

\section{AMPLIFIER DESIGN}

The discrete Raman amplifier design is shown in Fig. 1. It is built with two independent stages, each backward pumped with multiple semiconductor lasers, details shown in table 1.

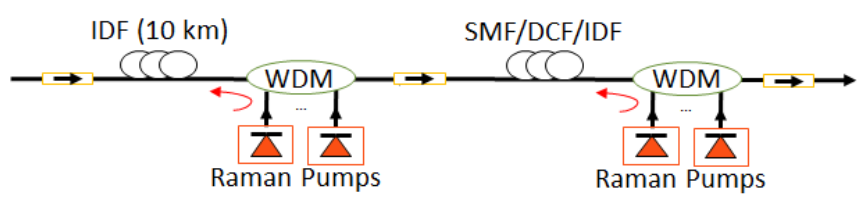

Fig. 1. Amplifier design: two backward pumped stages with the pump wavelengths in range of $1425-1491 \mathrm{~nm}$.

TABLE I

PUMP POWERS USED

\begin{tabular}{|c|c|c|c|c|c|}
\cline { 2 - 6 } \multicolumn{1}{c|}{} & $\begin{array}{c}1 \text { st } \\
\text { stage }\end{array}$ & \multicolumn{4}{c|}{$\begin{array}{c}\text { 2nd } \\
\text { stage }\end{array}$} \\
\hline $\begin{array}{c}\text { Pumps } \\
(\mathbf{n m})\end{array}$ & $\begin{array}{c}\text { IDF } \\
\mathbf{1 0 k m} \\
(\mathbf{m W})\end{array}$ & $\begin{array}{c}\text { SMF } \\
\mathbf{1 0 k m} \\
(\mathbf{m W})\end{array}$ & $\begin{array}{c}\text { IDF } \\
\mathbf{7 . 5 k m} \\
(\mathbf{m W})\end{array}$ & $\begin{array}{c}\text { DCF } \\
\mathbf{5 k m} \\
(\mathbf{m W})\end{array}$ & $\begin{array}{c}\mathbf{D C F} \\
\mathbf{1 0 k m} \\
(\mathbf{m W})\end{array}$ \\
\hline 1425 & 351 & 278 & 135 & 107 & 89 \\
\hline 1431 & 0 & 188 & 113 & 87 & 71 \\
\hline 1444 & 236 & 221 & 112 & 100 & 95 \\
\hline 1462 & 99 & 135 & 58 & 50 & 17 \\
\hline 1476 & 16 & 115 & 12 & 12 & 4 \\
\hline 1491 & 142 & 260 & 145 & 120 & 72 \\
\hline Total & $\mathbf{8 4 4}$ & $\mathbf{1 1 9 7}$ & $\mathbf{5 7 5}$ & $\mathbf{4 7 6}$ & $\mathbf{3 4 8}$ \\
\hline
\end{tabular}

For the first stage, we used $10 \mathrm{~km}$ of IDF $(0.23 \mathrm{~dB} / \mathrm{km}$ loss, $-44 \mathrm{ps} / \mathrm{nm} . \mathrm{km}$ dispersion at $1550 \mathrm{~nm}, \gamma=3.53 / \mathrm{W} . \mathrm{km})$. Four different fibre options were tested as the second stage: $10 \mathrm{~km}$ SMF $(0.2 \mathrm{~dB} / \mathrm{km}$ loss, $16.7 \mathrm{ps} / \mathrm{nm} . \mathrm{km}$ dispersion at $1550 \mathrm{~nm}$, $\gamma=1.3 / \mathrm{W} . \mathrm{km}), 5 \mathrm{~km}$ DCF $(0.67 \mathrm{~dB} / \mathrm{km}$ loss, $-97.6 \mathrm{ps} / \mathrm{nm} . \mathrm{km}$ dispersion at $1550 \mathrm{~nm}, \gamma=8.43 / \mathrm{W} . \mathrm{km}), 10 \mathrm{~km}$ DCF, and $7.5 \mathrm{~km}$ IDF. Figure 2 shows a performance comparison of all four schemes, each pumped so as to have a similar broadband average net gain (a) of $19.5 \mathrm{~dB}$ across $>70 \mathrm{~nm}$ from 1530 $1600 \mathrm{~nm}$. For this measurement, $\mathrm{C}$ and L band EDFA ASE was used, which was shaped with the help of wavelength selective switches (WSSs) to generate 24 channels (12 per band) from 192.5-195.8THz (1531.12-1557.36nm) in the C-band and 187.4-190.7THz (1572.06-1599.75nm) in the L-band, -20dBm power per channel and $300 \mathrm{GHz}$ spacing within each band [6].
As seen in Fig. 2b, the resulting noise figure (NF) was found to be quite similar, which is expected, given it is mainly dominated by the constant first stage. Figure $2 \mathrm{c}$ shows the average gain saturating faster for the more nonlinear fibres. This is a result of the difference in total pumping power and in pump-pump interactions, whereby for fibres with higher nonlinearity a more efficient pump energy transfer from lower to higher wavelengths was observed. IDF-SMF presented the highest saturated output power of $>22 \mathrm{dBm}$.

\section{NONLINEAR NOISE CHARACTERISATION USING THE FWM TECHNIQUE}

A FWM measurement [10] was used to characterise the performance of the four different DRA schemes with the setup shown in Fig. 3. Two tuneable $\mathrm{CW}$ lasers $\left(\mathrm{P}_{1}=\mathrm{P}_{2}=3 \mathrm{dBm}\right)$ were combined with the use of a 50/50 coupler and launched into a $93.4 \mathrm{~km}$ long SMF span, where the $19.5 \mathrm{~dB}$ loss was compensated by the DRA. The FWM efficiency was measured around $191.9 \mathrm{THz}$, where the frequency separation of the lasers $(\Delta \mathrm{f})$ was varied from $1 \mathrm{GHz}$ to $50 \mathrm{GHz}$, with the resulting FWM power $\left(\mathrm{P}_{\mathrm{FWM}}\right)$ recorded on a high resolution $(150 \mathrm{MHz})$ optical spectrum analyser (OSA).

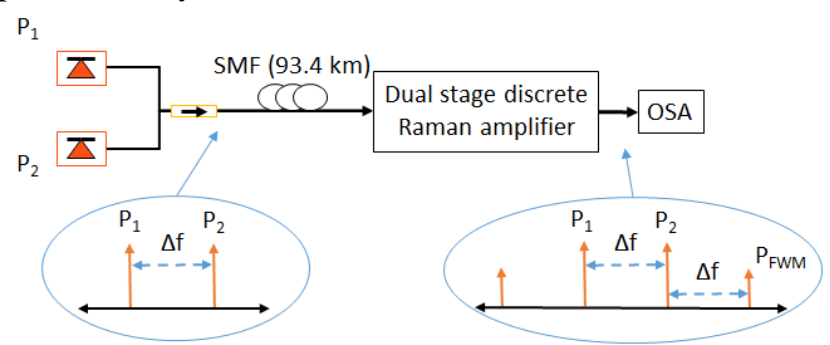

Fig. 3. FWM measurement setup.

As expected, the IDF-SMF configuration had the lowest FWM product power due to the bigger core area of the SMF and the resulting lower nonlinearity. At $1 \mathrm{GHz}$ frequency separation it was measured to be $-24.2 \mathrm{dBm}, 1.4 \mathrm{~dB}$ lower than both IDF-DCF5 $\mathrm{km}$ and IDF-IDF7.5km configurations and $3.2 \mathrm{~dB}$ lower than the IDF-DCF $10 \mathrm{~km}$ scheme. The integrated FWM power over $1 \mathrm{GHz}$ to $15 \mathrm{GHz}$ frequency separation was very similar for both IDF-IDF7.5km and IDF-DCF5 km cases, giving values of $-16.9 \mathrm{~dB}$ and $-17 \mathrm{~dB}$ respectively and placing them $1.8 \mathrm{~dB}$ above the IDF-SMF scheme $(-18.8 \mathrm{dBm})$ and $1.5 \mathrm{~dB}$ below the IDF-DCF10km setup (-15.5dBm).

IDF $10 \mathrm{~km}-\mathrm{SMF} 10 \mathrm{~km} \quad \square \quad$ IDF $10 \mathrm{~km}-\mathrm{DCF} 5 \mathrm{~km}$
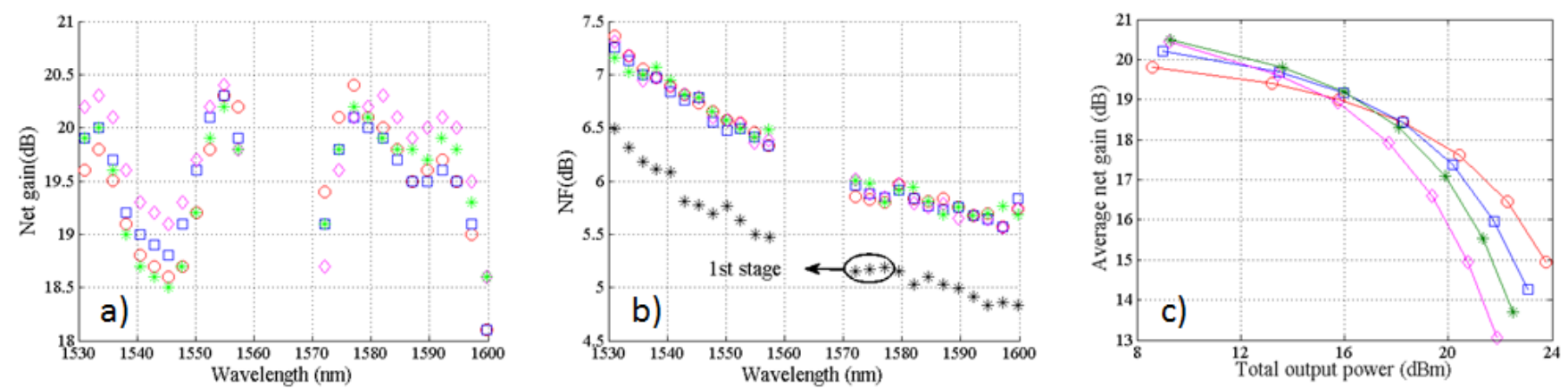

Fig. 2. Performance comparison of different DRA schemes: (a) net gain vs wavelength, (b) NF vs wavelength, (c) average net gain over 70nm bandwidth vs total output power. 


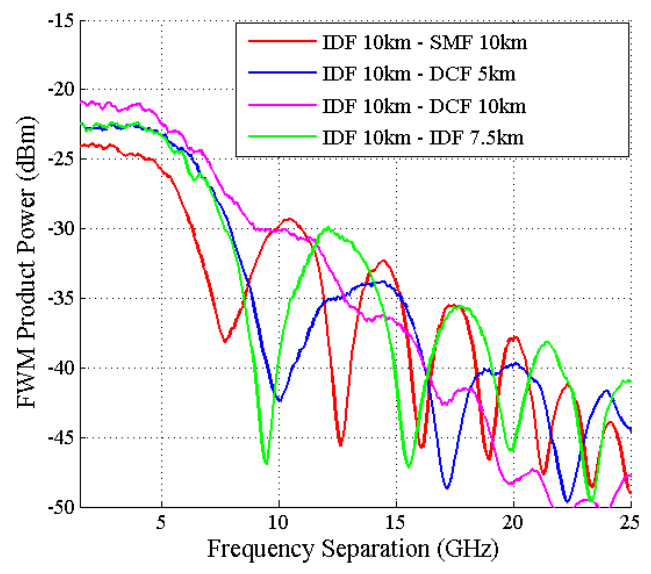

Fig. 4. Experimental product power as a function of the frequency separation between two CW lasers ( $3 \mathrm{dBm}$ each).

\section{NONLINEAR NOISE CHARACTERISATION USING TRANSMISSION SETUP}

The performance of every dual-stage DRA scheme was characterised through a WDM coherent transmission experiment inside a recirculating loop as shown in Fig. 5 [6]. Five 100GHz spaced (193.8 - 194.2THz), 120Gb/s DP-QPSK signals were generated using an IQ modulator and a polarization multiplexer (PolMux). A 30Gb/s, $2^{31}-1$ long pseudo random binary sequence (PRBS) was applied to each I and $\mathrm{Q}$ modulator arms with a 18 bits relative delay. The resulting $5 \times 30$ GBaud PM-QPSK signals were then amplified by a $23 \mathrm{dBm}$ output power EDFA and a variable attenuator was used to launch -5.7 to $4.3 \mathrm{dBm} /$ channel into the SMF fibre span while keeping the input OSNR fixed.

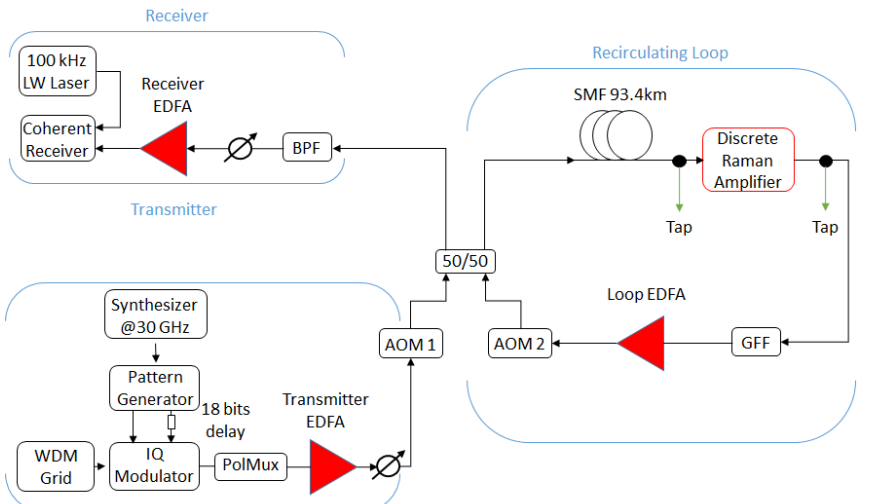

Fig. 5. Transmission setup with the use of a recirculating loop.

The loop itself consisted of a 93.4km SMF transmission span (19.5dB loss) followed by the dual stage DRA under test. A pair of 95/5 taps were used before and after the dual stage DRA to monitor the gain and adjust the pumping power to maintain a $19.5 \mathrm{~dB}$ gain that was independent of input signal power. An EDFA was used to compensate the additional $\sim 15 \mathrm{~dB}$ loop losses from the gain flattening filter (GFF), 3dB coupler, acousto-optic modulator (AOM), taps etc.

The receiver chain consisted of a tuneable band pass filter (BPF) followed by an attenuator and an EDFA to provide constant power into a polarization diverse coherent receiver, where the signal was mixed with a $100 \mathrm{kHz}$ linewidth local oscillator and captured using an $80 \mathrm{GSa} / \mathrm{s}, 36 \mathrm{GHz}$ real-time oscilloscope. The recorded data was then processed using offline digital signal processing (DSP), where the $\mathrm{Q}^{2}$ was derived from the bit error ratio (BER) [11].

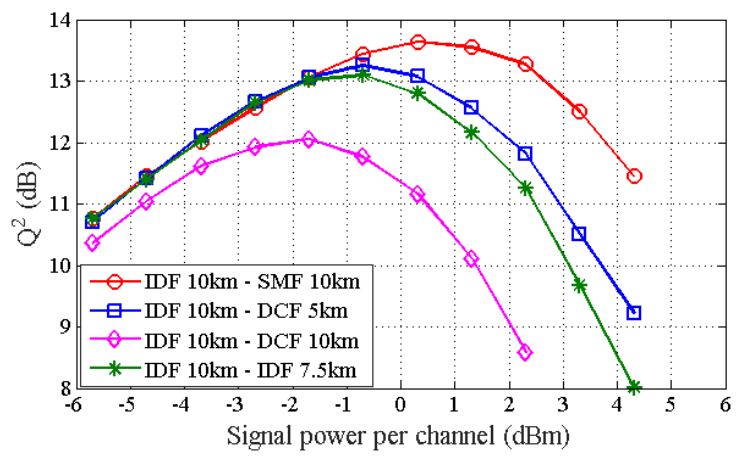

Fig. 6. $Q^{2}$ vs signal power per channel for different dual-stage DRA schemes for 10 spans of $93.4 \mathrm{~km}$ transmission length for $194 \mathrm{THz}$ channel.

Figure 6 shows the $\mathrm{Q}^{2}$ vs signal power per channel for the centre WDM signal $(194 \mathrm{THz})$ at $10 \times 93.4 \mathrm{~km}$ transmission distance. The IDF-SMF based DRA showed the maximum $\mathrm{Q}^{2}$ factor of $13.6 \mathrm{~dB}$, with the IDF-DCF5 km and IDF-IDF7.5km reaching $13.2 \mathrm{~dB}$ and $13.1 \mathrm{~dB}$ respectively. The IDF-DCF10km configuration, which is the most affected by nonlinear effects, had a maximum $\mathrm{Q}^{2}$ factor of $12 \mathrm{~dB}$ with its optimum launch power $2 \mathrm{~dB}$ below the IDF-SMF configuration. The differences in the nonlinear regime match the predictions of FWM product measurements presented in Fig. 4. The nonlinear impairments increase with signal power and reach the highest measured penalty of $2.2 \mathrm{~dB}$ for IDF-DCF5 km, $3.4 \mathrm{~dB}$ for IDF-IDF and $4.7 \mathrm{~dB}$ for IDF-DCF $10 \mathrm{~km}$ with respect to the IDF-SMF configuration. In the linear regime, which is limited by ASE amplifier noise, all the schemes showed similar performance which can be expected from similar NF measured in Fig. 2(b).

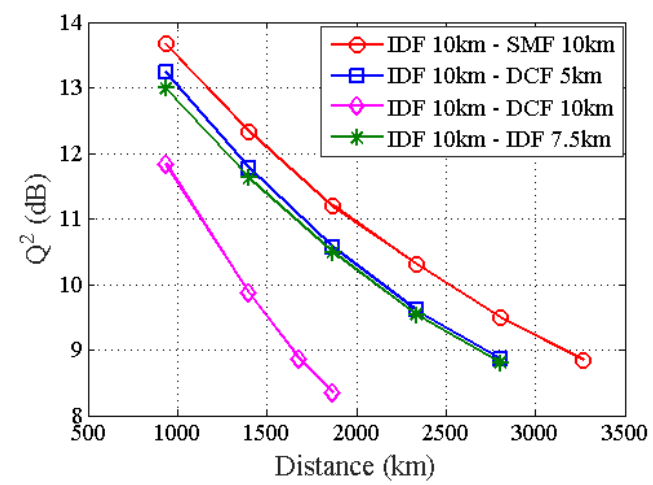

Fig. 7. $Q^{2}$ vs transmission distance for different dual-stage DRA schemes.

All four DRA configurations were tested for the maximum possible transmission distance using the optimum signal launch power and assuming a $\mathrm{Q}^{2}$ FEC threshold of $8.5 \mathrm{~dB}$ (corresponding to BER $3.8 \times 10^{-3}$ ), results of which can be seen in Fig. 7. Using IDF-SMF, which had the highest optimum launch power, it was possible to reach 35 recirculations $(3269 \mathrm{~km})$ with a $\mathrm{Q}^{2}$ of $8.8 \mathrm{~dB}$. This was 5 recirculations $(467 \mathrm{~km})$ more than in the IDF-DCF5 km and IDF-IDF7.5km configurations for the same $\mathrm{Q}^{2}$. With the IDF-DCF10km it was possible to reach only half of the IDF-SMF distance $(1681 \mathrm{~km})$. 


\section{DISCUSSION}

The average net gain was kept similar for all four DRA schemes at $\sim 19.5 \mathrm{~dB}$ and their NF was found to be similar to each other (Fig. 2a and Fig. 2b), which is readily understood given that the NF of dual-stage amplifiers is mainly dominated by the first stage NF. The maximum NF of the $1^{\text {st }}$ stage IDF $10 \mathrm{~km}$ was $6.5 \mathrm{~dB}$ and a $<1 \mathrm{~dB} \mathrm{NF}$ increase was observed after adding the second stage. The NF tilt of $\angle 2 \mathrm{~dB}$ is due to backward pumping configuration and could be reduced by using bidirectional pumping [12]. Pump-pump interactions are an important issue for broadband amplification. In fibres with higher nonlinearity (IDF, DCF) a stronger energy transfer from lower to higher pumping wavelengths than in the case of SMF results in a different distribution of pumping powers required for each case. This effect is modified when the amplifier is driven into saturation, which further changes the power distribution and may result in additional gain flatness distortion. For the measurements of gain, NF and saturation output power, the pumps were kept fixed, whereas for the nonlinear characterisation the pumps were adjusted to keep the gain and amplifier output power unchanged so that saturation effects wouldn't affect the results. As the DRA schemes described above have different saturation points (see Fig. 2(c)), a fair comparison of nonlinear penalties among different configurations requires fixed gain to ensure the same signal power profiles for each case.

Although IDF-SMF can be intuitively identified as the scheme least affected by nonlinearities, the FWM product power measurements gave us an insight on the quantifiable amount of the difference between all the DRA architectures. As the Kerr-induced nonlinearities accumulate over increased transmission distance, 10 spans of $93.4 \mathrm{~km}$ SMF fibre were chosen to perform the characterisation of nonlinear performance of the dual stage DRAs. As mentioned before, the gain was kept constant for all the input powers to prevent saturation effects from masking the impact of DRA fibre nonlinearity. An attenuator was used before the receiver EDFA to keep its NF constant for the same reason as mentioned above.

The transmission results in Fig. 6 achieved with our DRAs are qualitatively similar to those of SMF links with EDFAs [13], but with reduced performance. The additional nonlinear impairments due to high power signal propagation in the nonlinear DRA fibre depend on the exact signal power profile [2] and the dispersion map introduced by the DRA, and can be predicted by the GN-model [13]. Using SMF versus DCF or IDF for the second stage not only reduces the nonlinear impairments of a DRA but also increases the total dispersion of the link, which is beneficial for the signal transmission [13]. These nonlinear impacts are most important over long distances, but in metro networks where distances are relatively short and capacity growth currently strongest, the received OSNR is paramount as it determines the useable higher-order modulation format [14]. We anticipate the increased bandwidth of systems based on DRAs with tolerable nonlinear impact, as described here, could readily increase network capacity.

\section{CONCLUSION}

We have characterized a dual stage IDF-SMF design of discrete broadband Raman amplifier in terms of linear and nonlinear noise performance. We have shown that while the linear noise figure is mainly dominated by the first stage, using SMF in the second stage can significantly reduce the nonlinear noise induced penalties. Our $19.5 \mathrm{~dB}$ broadband $(>70 \mathrm{~nm})$ net gain amplifier was proven to generate significantly lower FWM product power, which gave a superior performance in the nonlinear regime compared with other tested DRA architectures, and resulted in an up to $2 \mathrm{~dB}$ optimum launch power improvement with a $1.6 \mathrm{~dB}$ increase in optimum $\mathrm{Q}^{2}$ factor at 10 spans of $93.4 \mathrm{~km}$ SMF of transmission. With the proposed IDF-SMF architecture we measured a $\mathrm{Q}^{2}$ of $8.8 \mathrm{~dB}$ after $3269 \mathrm{~km}$ transmission, which is $>460 \mathrm{~km}$ further than for the second best tested (IDF-IDF) configuration and twice as far as for the worst performing one (IDF-DCF).

\section{REFERENCES}

[1] M. N. Islam, "Raman amplifiers for telecommunications," in IEEE Journal of Selected Topics in Quantum Electronics, vol. 8, no. 3, pp. 548559, May/Jun 2002.

[2] S. Namiki and Y. Emori, "Ultrabroad-band Raman amplifiers pumped and gain-equalized by wavelength-division-multiplexed high-power laser diodes," in IEEE Journal of Selected Topics in Quantum Electronics, vol. 7, no. 1, pp. 3-16, Jan/Feb 2001.

[3] Y. Emori and S. Namiki, "100 nm bandwidth flat gain Raman amplifiers pumped and gain-equalized by 12-wavelength-channel WDM high power laser diodes," OFC/IOOC Technical Digest. Optical Fiber Communication Conference, 1999, and the International Conference on Integrated Optics and Optical Fiber Communication, San Diego, CA, USA, 1999, pp. PD19/1-PD19/3 Suppl..

[4] L. E. Nelson, X. Zhou, B. Zhu, M. F. Yan, P. W. Wisk and P. D. Magill, "All-Raman-Amplified, $73 \mathrm{~nm}$ Seamless Band Transmission of $9 \mathrm{~Tb} / \mathrm{s}$ Over $6000 \mathrm{~km}$ of Fiber," in IEEE Photonics Technology Letters, vol. 26, no. 3, pp. 242-245, Feb.1, 2014.

[5] M. A. Iqbal et al., "Performance characterization of high gain, high output power and low noise cascaded broadband discrete Raman amplifiers," 2017 19th International Conference on Transparent Optical Networks (ICTON), Girona, 2017, pp. 1-4.

[6] L. Krzczanowicz, M. Iqbal, I. Phillips, M. Tan, P. Skvortcov, P. Harper, and W. Forysiak, "Low transmission penalty dual-stage broadband discrete Raman amplifier," in Optics Express, vol. 26, pp. 7091-7097, 2018.

[7] Z. Zang, "Analysis of optical switching in a Yb 3+-doped fiber Bragg grating by using self-phase modulation and cross-phase modulation," in Applied Optics, vol. 51, no. 16, pp.3424-3430, 2012.

[8] D. Hamoir, J. Boniort, L. Gasca, and D. Bayart, "Optimized, two-stage architecture for Raman amplifiers," in Optical Amplifiers and Their Applications, A. Mecozzi, M. Shimizu, and J. Zyskind, eds., Vol. 44 of OSA Trends in Optics and Photonics (Optical Society of America, 2000), paper OMD8.

[9] S. A. E. Lewis, S. V. Chernikov and J. R. Taylor, "Characterization of double Rayleigh scatter noise in Raman amplifiers," in IEEE Photonics Technology Letters, vol. 12, no. 5, pp. 528-530, May 2000.

[10] M. A. Z. Al-Khateeb, M. Tan, M. A. Iqbal, M. McCarthy, P. Harper and A. D. Ellis, "Four wave mixing in distributed Raman amplified optical transmission systems," 2016 IEEE Photonics Conference (IPC), Waikoloa, HI, 2016, pp. 795-796.

[11] I. D. Phillips et al., "Exceeding the nonlinear-Shannon limit using raman laser based amplification and optical phase conjugation," OFC 2014, San Francisco, CA, 2014, pp. 1-3.

[12] S. Kado, Y. Emori and S. Namiki, "Gain and noise tilt control in multiwavelength bi-directionally pumped Raman amplifier," Optical Fiber Communication Conference and Exhibit, 2002, pp. 62-63.

[13] P. Poggiolini, A. Carena, V. Curri, G. Bosco and F. Forghieri, "Analytical Modeling of Nonlinear Propagation in Uncompensated Optical Transmission Links," in IEEE Photonics Technology Letters, vol. 23, no. 11, pp. 742-744, June 1, 2011.

[14] P. J. Winzer, "High-Spectral-Efficiency Optical Modulation Formats," in Journal of Lightwave Technology, vol. 30, no. 24, pp. 3824-3835, Dec.15, 2012. 